\title{
Donor infection with multidrug resistant organisms: Should we change our approach to perioperative prophylaxis?
}

Transmission of disease from donor to recipient remains an inevitable consequence of solid organ transplantation. In fact, the intentional transmission of viruses such as cytomegalovirus is routine; management strategies reduce the negative consequences of these transmissions. Unexpected transmission of disease is much less common, complicating $<1 \%$ of organ transplants. Nonetheless, consequences can be severe with significant recipient morbidity and mortality and the potential to reduce public trust in the safety of the organ procurement system.

Much of the regulatory attention regarding donor-derived disease has focused on blood borne viruses, particularly-in light of the opioid epidemic-hepatitis C virus (HCV). More sensitive testing methods combined with direct acting antivirals that cure posttransplant HCV have reduced the risk and consequence of donor transmission of HCV. What then should the transplant community prioritize in decreasing the impact of donor disease transmission? One emerging and poorly studied area is donor infection with multidrug-resistant organisms (MDRO). The World Health Organization cites antibiotic resistance as "one of the biggest threats to global health, food, and security" and that "new resistance mechanisms are emerging and spreading globally, threatening our ability to treat common diseases." ${ }^{1}$ According to the Centers for Disease Control and Prevention, 2 million infections with MDROs resulting in 23000 deaths occur yearly in the United States. ${ }^{2}$ In some circumstances, no reliably effective antibiotics are available to treat these organisms.

The published literature on the impact of donor-derived infection with MDROs is limited, but transmission of infection from donors infected at various sites (blood, sputum, preservative fluid, peritoneal fluid) with significant recipient morbidity has been described. ${ }^{3-5}$ In a large cases series from Italy, 30 recipients were exposed to donors infected with carbapenem-resistant gram negative bacteria. In 14 of these 30 recipients, the donor had either bacteremia or infection of the transplanted organ. Four of six exposed recipients not promptly receiving active antimicrobial therapy developed clinical infection or colonization, and one died. Interestingly, the eight recipients effectively treated for at least 7 days did not develop donor-derived infection. ${ }^{4}$

Organs from deceased donors have many of the traditional risk factors for infection with MDROs including prolonged hospitalization, receipt of antimicrobials, treatment in an intensive care unit, and mechanical ventilation. When culture and sensitivity information is available preprocurement (eg, isolation of multidrug-resistant Acinetobacter from a tracheal aspirate isolated a few days prior to lung procurement) tailored antibiotics can be administered to the recipient, reducing the risk of infection. The more common scenario, however, is that hospital cultures (obtained as part of routine care) or those performed specifically as part of the donation evaluation process become available after transplantation. In this issue, Anesi and colleagues studied whether specific donor characteristics predicted donor infection ("active" infection not distinguished from colonization) with MDROs. ${ }^{6}$ The investigators reviewed deceased donors who donated to one of 4 Philadelphia transplant centers and were evaluated by the local organ procurement organization (OPO). Time to donor isolation of an MDRO was the primary outcome, and donor characteristics including antibiotic treatment were used to identify risk factors for MDRO infection.

The most important finding was that MDRO infection rates increased with length of stay with $20 \%$ of donors infected by hospital day 10. Methicillin-resistant Staph aureus (MRSA) was the most frequent pathogen, and most infections involved the respiratory tract with only 5 (1\%) bloodstream infections. Important risk factors for MDRO infection on multivariable analysis included HCV viremia (largely for MRSA), dialysis, receipt of narrow-spectrum gram negative antibiotics, and asphyxiation (for resistant Enterobacteriaceae). Interestingly the T4 protocol (which uses glucocorticoids and extensive central vascular access) was also associated with MRSA infection.

How can these findings be used to reduce donor-derived infection? Although the rates of MDRO donor colonization are alarming, most of the cultures are from respiratory samples that-although they might indicate colonization at other sites-have not been shown to require targeted interventions in nonlung recipients, and certainly universally broadening antimicrobial prophylaxis is likely to worsen the MDRO problem. Rather, these findings may represent an important early step toward a targeted approach where a combination of factors (eg, prolonged duration of hospitalization, colonization with MDRO organisms at multiple sites, prolonged antimicrobial treatment) may identify donors at a high enough risk of MDRO infection to justify enhanced perioperative prophylaxis. Such an approach would be complex, would need to account for local differences in MDRO ecology, and distinguish between gram positive and gram negative MDROs because treatment would differ. Importantly, given the risk of creating further resistance, a better understanding of recipient outcomes associated with donor MDRO infection is required before enhanced prophylaxis can be recommended. Although the Anesi study is an excellent beginning, in addition to assessing 
recipient outcomes, future studies should involve multiple OPOs as local differences in resistance patterns and MDROs of greatest concern would be expected. It is hoped that research in this area will help us make the most informed choice when faced with the decision to accept or decline an organ offer from a donor infected with an MDRO.

\section{DISCLOSURE}

The author of this manuscript has no conflicts of interest to disclose as described by the American Journal of Transplantation.

Daniel R. Kaul

Department of Internal Medicine, Division of Infectious Diseases, University of Michigan, Ann Arbor, Michigan

Correspondence

Daniel R. Kaul Email: kauld@umich.edu

\section{REFERENCES}

1. About AMR. http://www.euro.who.int/en/health-topics/diseaseprevention/antimicrobial-resistance/about-amr. Accessed June 1, 2019.

2. Centers for Disease Control and Prevention. Antibiotic/Antimicrobial Resistance (AR/AMR). https://www.cdc.gov/drugresistance/biggest_ threats.html. Accessed June 1, 2019.

3. Giani T, Conte V, Mandala S, D'Andrea MM, Luzzaro F, Conaldi PG, et al. Cross-infection of solid organ transplant recipients by a multidrug-resistant Klebsiella pneumoniae isolate producing the OXA-48 carbapenemase, likely derived from a multiorgan donor. J Clin Microbiol. 2014;52(7):2702-2705.

4. Mularoni A, Bertani A, Vizzini G, Gona F, Campanella M, Spada M, et al. Outcome of Transplantation using organs from donors infected or colonized with carbapenem-resistant gram-negative bacteria. Am J Transplant. 2015;15(10):2674-2682.

5. Wendt JM, Kaul D, Limbago BM, Ramesh M, Cohle S, Denison AM, et al. Transmission of methicillin-resistant Staphylococcus aureus infection through solid organ transplantation: confirmation via whole genome sequencing. Am J Transplant. 2014;14(11):2633-2639.

6. Anesi JA, Blumberg EA, Han JH, Lee DH, Clauss H, Climaco A, et al. Risk factors for multidrug-resistant organisms among deceased organ donors. [published online ahead of print June 4, 2019] Am J Transplant. https://doi.org/10.1111/ajt.15488. 